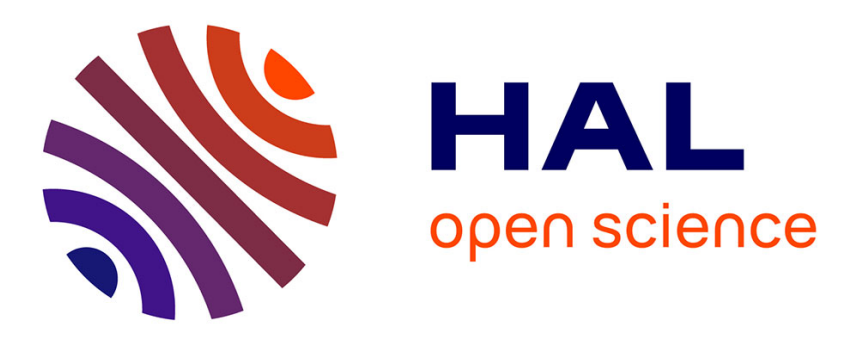

\title{
Investigation on 3D high frame rate imaging with motion compensation (MoCo)
}

Sébastien Salles, Francois Varray, Damien Garcia, Barbara Nicolas, Herve

Liebgott

\section{- To cite this version:}

Sébastien Salles, Francois Varray, Damien Garcia, Barbara Nicolas, Herve Liebgott. Investigation on 3D high frame rate imaging with motion compensation (MoCo). IEEE International Ultrasonics Symposium, Oct 2019, Glasgow, United Kingdom. hal-02352623

\section{HAL Id: hal-02352623 \\ https://hal.science/hal-02352623}

Submitted on 6 Nov 2019

HAL is a multi-disciplinary open access archive for the deposit and dissemination of scientific research documents, whether they are published or not. The documents may come from teaching and research institutions in France or abroad, or from public or private research centers.
L'archive ouverte pluridisciplinaire HAL, est destinée au dépôt et à la diffusion de documents scientifiques de niveau recherche, publiés ou non, émanant des établissements d'enseignement et de recherche français ou étrangers, des laboratoires publics ou privés. 


\section{Investigation on 3D high frame rate imaging with motion compensation (MoCo)}

\author{
Sebastien Salles \\ Univ Lyon, INSA - Lyon, Université \\ Claude Bernard Lyon 1, UJM-Saint \\ Etienne, CNRS, Inserm, CREATIS UMR \\ 5220, U1206, F - 69621, LYON, France,
}

\author{
Barbara Nicolas \\ Univ Lyon, INSA - Lyon, Université \\ Claude Bernard Lyon 1, UJM-Saint \\ Etienne, CNRS, Inserm, CREATIS UMR \\ 5220, U1206, F - 69621, LYON, France
}

\author{
Francois Varray \\ Univ Lyon, INSA - Lyon, Université \\ Claude Bernard Lyon 1, UJM-Saint \\ Etienne, CNRS, Inserm, CREATIS UMR \\ 5220, U1206, F - 69621, LYON, France
}

\author{
Hervé Liebgott \\ Univ Lyon, INSA - Lyon, Université \\ Claude Bernard Lyon 1, UJM-Saint \\ Etienne, CNRS, Inserm, CREATIS UMR \\ 5220, U1206, F - 69621, LYON, France
}

\author{
Damien Garcia \\ Univ Lyon, INSA - Lyon, Université \\ Claude Bernard Lyon 1, UJM-Saint \\ Etienne, CNRS, Inserm, CREATIS UMR \\ 5220, U1206, F - 69621, LYON, France
}

\begin{abstract}
Cardiac high-frame-rate (HFR) imaging has shown promise in bringing new diagnostic information. The development of advanced 3D-HFR echocardiographic techniques, with the aim of providing high image quality, has become a key research focus. Methods such as Steered Diverging Waves and Sub-Aperture Diverging Waves have been already extended to 3D. Because phase delays due to large tissue displacements can blur the compound images, 3D Motion compensation (MoCo) approaches have recently been introduced and integrated in the compounding process. In this in silico work, we were interested in investigating the influence of the MoCo approach on three different diverging waves strategies, each with three different configurations of virtual sources. First, the locations of the virtual sources were formalized according to the chosen diverging wave strategy and the diverging wave opening angle. Then, the nine approaches were compared quantitatively by estimating the CNR and CR. The results confirmed that MoCo increased the CNR andCR for each case.
\end{abstract}

Keywords - Ultrafast Imaging, Motion Compensation, Diverging Waves, Cardiac imaging, 3D Echocardiography

\section{INTRODUCTION}

In the past few years, research has focused on the development of new acquisition schemes able to perform 3$\mathrm{D}$ ultrasound imaging with higher temporal resolution than conventional approaches available on commercial scanners. The most promising solution is the 3-D extension of a radarbased technique called synthetic aperture. Synthetic aperture imaging consists of coherently combining images with low spatial resolution at a frame rate equal to the pulse repetition frequency (PRF) into one image with high spatial resolution. This technique makes it possible to obtain high-frame-rate imaging ( 500 to $10000 \mathrm{frame} / \mathrm{s}$ ) while keeping a high enough image quality. Naturally, a compromise must be found between frame rate and image quality which will depend on the targeted applications. Low resolution can be achieved in different ways, for example by using plane or spherical waves. . Tanter and Fink's group introduced the term coherent compounding, which uses multiple plane waves emitted from different angles before being summed to form a high-resolution image [1]. Jensen's group used spherical waves to obtain high-frame-rate vector velocity imaging [2]. Both techniques use synthetic aperture imaging, where low-resolution images from different insonification angles are added together. A third way to obtain high-framerate imaging is the multi-line transmit (MLT) approach, which combines several focused beams into a single image [3]. Both techniques have been extended in 3-D for multiple applications, such as vascular and cardiac imaging [4]-[6]. Synthetic aperture can be achieved with two different acquisition schemes. Both schemes include the placement of a number of virtual sources behind the probe. The first approach consists in transmitting several diverging waves, with different steering angles, by keeping the same subaperture for all transmissions. The second approach consists in transmitting several diverging waves with the same steering angles, by sliding the sub-aperture according the position of virtual sources.

For specific applications, such as cardiac imaging, the compounding process may suffer from phase delays due to large tissue displacements between the first and last transmission. Phase delays between the transmissions lead to an incoherent compounding, which leads to the deterioration of the image quality. Motion Compensation (MoCo) approaches have recently been introduced to compensate for the tissue motion within the compounding process. Different techniques were tested in high-frame-rate 2-D echocardiography with synthetic aperture [7]-[9]. More recently, 3-D MoCo approach was tested in simulation, by comparing the results obtained with synthetic aperture (with sliding sub-aperture) and MLT approach [10]. Moreover, the feasibility of 3-D MoCo has been experimentally demonstrated [11]. This work aims at investigating the influence of the MoCo approach on three different synthetic 
aperture strategies with three differents configuration of virtual sources in simulation.

\section{MATERIAL AND METHOD}

\section{A. 3D Diverging waves strategies}

Three different diverging wave strategies (S-1,.., S-3, Fig 1) were considered in this work. For each strategy, three different configurations of virtual sources were tested [C$1, \ldots \mathrm{C}-3]$. The two transverse coordinates of the virtual sources, $v s_{x}$ and $v s_{y}$ were defined as follows:

$$
\begin{aligned}
& v s_{x}(i, j)=\frac{N_{x} \times(1-D)}{2 \times D} \times \text { pitch }_{x}+(i-1) \times \text { pitch }_{x}, \\
& i:\{1,2, \ldots \sqrt{\mathrm{Ns}}\}, j:\{1,2, \ldots \sqrt{\mathrm{Ns}}\}
\end{aligned}
$$

and

$$
\begin{aligned}
& v s_{y}(i, j)=\frac{N_{y} \times(1-D)}{2 \times D} \times \text { pitch }_{y}+(\mathrm{j}-1) \times \text { Vpitch }_{y}, \\
& i:\{1,2, \ldots \sqrt{\mathrm{Ns}}\}, j:\{1,2, \ldots \sqrt{\mathrm{Ns}}\}
\end{aligned}
$$

with

$$
\text { Vpitch }_{x, y}=N_{x, y} \times \frac{(D-1)}{D \times(\sqrt{\mathrm{Ns}}-1)} \times \text { pitch }_{x, y}
$$

Where $N_{s}$ is the number of virtual sources, $N_{x, y}$ is the number of elements in each direction, and $\mathrm{D}$ a constant corresponding to the ratio between the size of the chosen subaperture and the size of the full aperture. The three different virtual source configurations were defined according to the values of $\mathrm{D}(2,3,4)$.

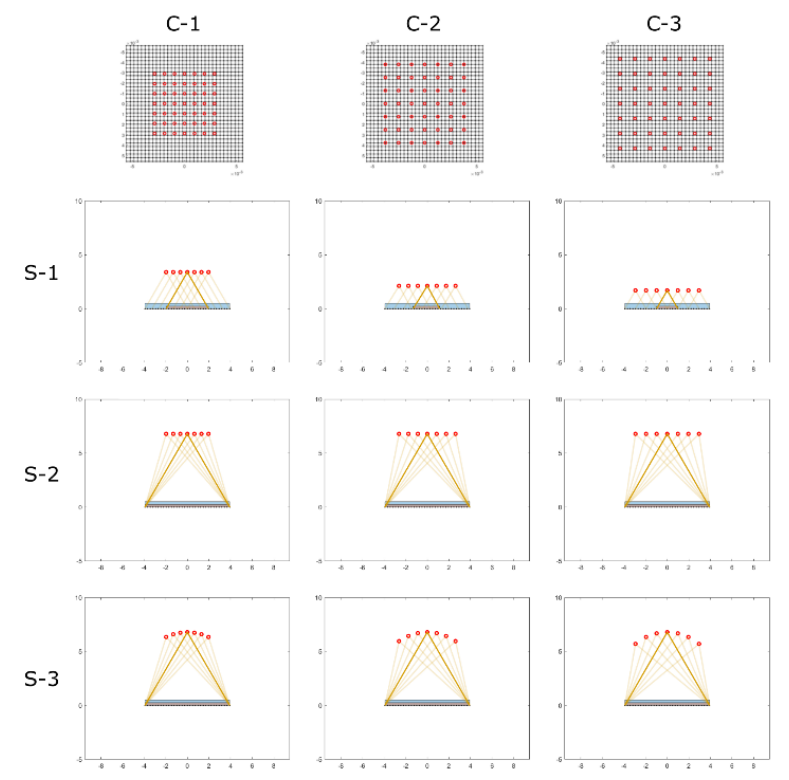

Fig 1: 2D representation of the nine different transmission strategies.

The first strategy (S-1) consists in transmitting 3D diverging waves with a sub-aperture sliding according the position of the virtual sources [10]. The axial position of the virtual sources $v S_{z}$ was defined as follows:

$$
S-1 \quad: \quad v S_{z}=\frac{N_{x, y} \times \text { pitch }_{x, y}}{2 \times D \times \tan (\alpha / 2)}
$$

where $\alpha$ is the chosen opening angle of the diverging wave.

The second strategy (S-2) consists in transmitting 3D steered diverging waves with the full aperture. The axial position of the virtual sources $v s_{z}$ was defined as follows:

$$
S-2 \quad: \quad v s_{z}=\frac{N_{x, y} \times \text { pitch }_{x, y}}{2 \times \tan \left(\alpha_{m} / 2\right)}
$$

where $\alpha_{m}$ is the chosen opening angle of the diverging wave, at the probe center. With this strategy, the virtual sources are placed on a plane parallel to the probe. By doing so, the opening angles vary slightly from one transmission to another.

The third strategy (S-3) also consists in transmitting 3D steered diverging waves with the full aperture. In contrary to S-2, the virtual sources are placed on a spherical shaped surface in order to keep the opening angle constant for each transmission. $v S_{z}$ was defined as follow:

$$
S-3 \quad: \quad v s_{z}(i, j)=\sqrt{\frac{\pi \times\left(N_{x, y} \times p i t c h\right)^{2}}{4 \times \alpha}-v s_{x}{ }^{2}(i, j)-v s_{y}{ }^{2}(i, j)}
$$

where $\alpha$ is the chosen opening angle of the diverging waves.

\section{B. Proposed 3D MoCo strategy}

A "round-trip" scan sequence was achieved in order to perform MoCo (Fig. 2). The virtual sources (i.e., the black spots) are sequentially activated in a specific sequence from the start point to the endpoint, and then they are transmitted back from the endpoint to the start point. Hence, 50 transmissions were computed to reconstruct one volume.
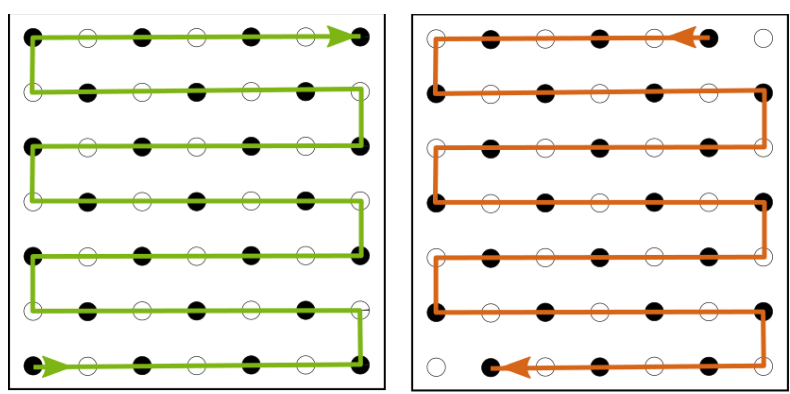

Fig. 2: MOCO strategy. The Green arrow indicates the forward path, and the red arrow indicates the backward path. The black spots indicate the activated virtual sources in the round-trip scan sequence. 
The 3-D motion compensation was performed by combining tissue Doppler imaging (TDI) with the transmit sequence [7].

$$
\hat{V}=\frac{\angle \hat{R}_{x}(1) \times v N y q}{\pi},
$$

where $\angle \hat{R}_{x}(1)$ represents the phase angle of the spatiotemporal filtered autocorrelation with lag 1 .

\section{Simulation Setup}

All simulations were carried out with Field II [12], [13]. A matrix array of 32-by-32 elements with a center frequency of $3 \mathrm{MHz}$ was used. In the beamforming process, the pulse repetition frequency (PRF) was set to $5 \mathrm{kHz}$. 2D Hanning window was applied in transmit. The imaging object was a $30 \mathrm{~mm} \times 30 \mathrm{~mm} \times 30 \mathrm{~mm}$ (azimuth $\times$ elevation $\times$ axial) cubic phantom containing a spherical cystic region with a radius of $10 \mathrm{~mm}$. The scattering amplitudes were randomly distributed between 0 and 1 following a uniform distribution outside the cystic region and set to 0 inside the cystic region. To investigate the performance of the proposed MoCo strategies, the phantom was moved in the axial direction at a speed of $10 \mathrm{~cm} / \mathrm{s}$.

\section{a. Without motion compensation}
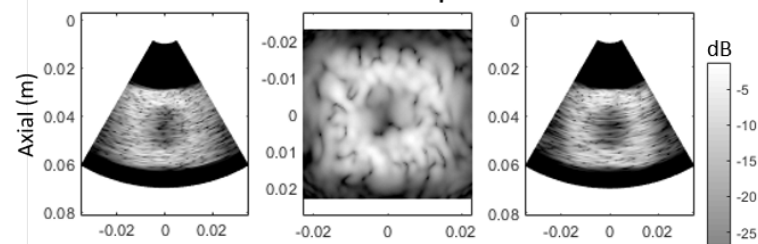

b. With motion compensation
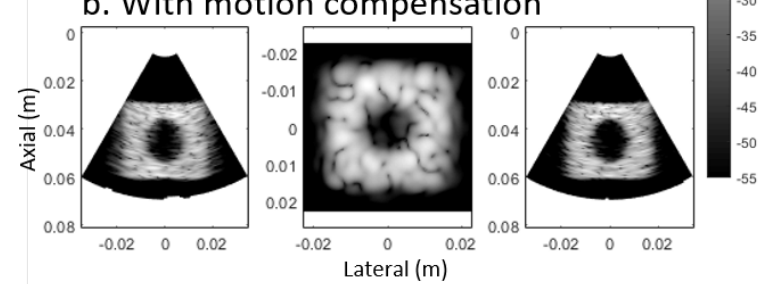

Fig. 3: Images obtained with the diverging waves strategy $\mathrm{S}-1$ and the $\mathrm{C}-1$ virtual sources configuration in simulation.

The metrics used to quantitavely evaluate the improvements of the proposed 3-D MoCo are the contrast ratio $(\mathrm{CR})$ and the contrast-to-noise ratio (CNR):

$$
\begin{aligned}
& C R=\gamma_{\text {out }}-\gamma_{\text {in }} \\
& C R=20 \times \log 10\left(\frac{\gamma_{\text {out }}-\gamma_{\text {in }}}{\sqrt{\sigma_{\text {out }}^{2}+\sigma_{\text {in }}^{2}}}\right)
\end{aligned}
$$

where $\gamma_{\text {out }}$ and $\gamma_{\text {in }}$ were the mean image data (after logcompression) outside and inside the cystic regions,

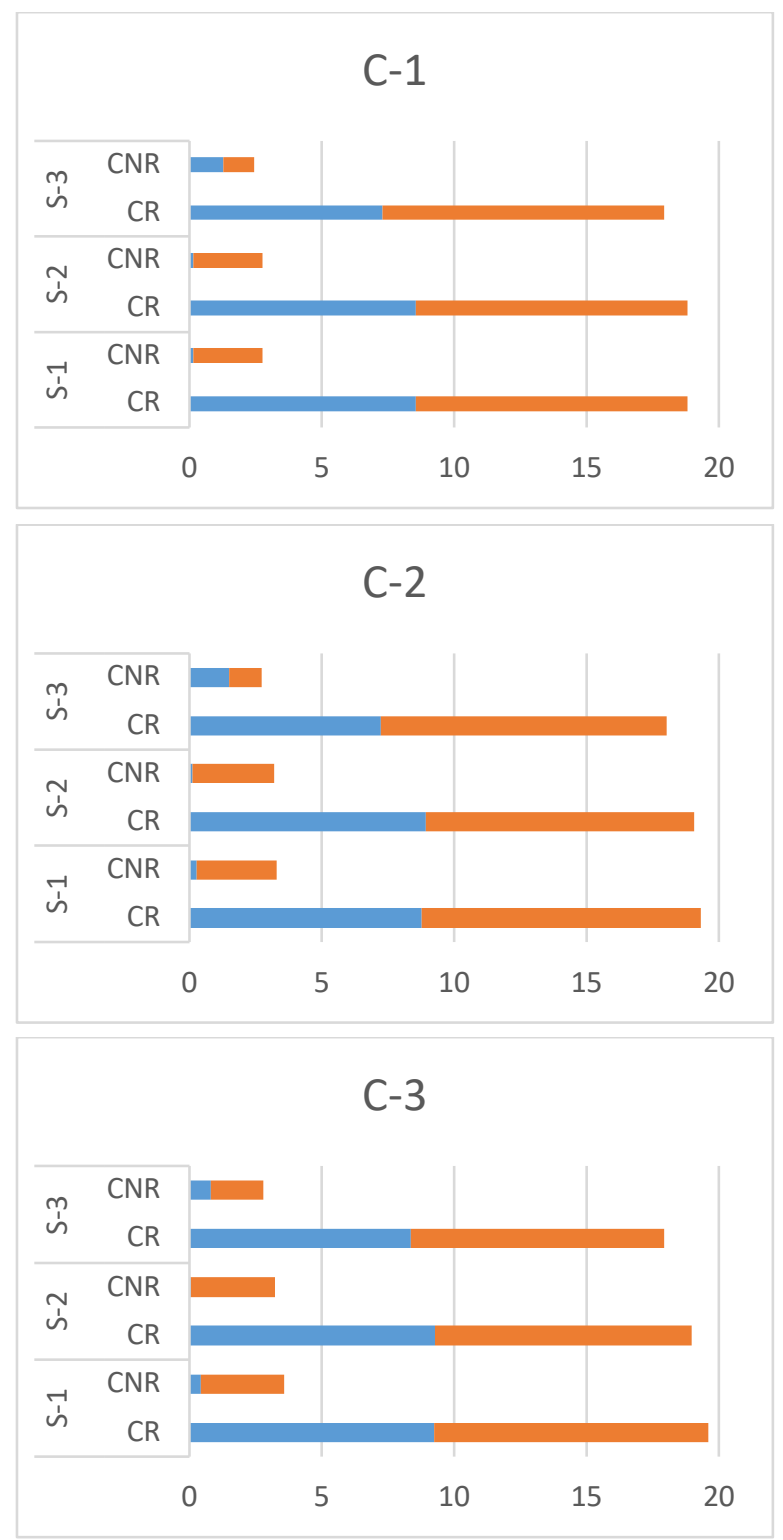

Fig. 4 Quantitative CNR and CR for the three virtual sources configuration and the three diverging waves strategies. The bars in blue/red colors indicate the results without/with 3D MoCo, respectively

respectively. Moreover, $\sigma_{\text {out }}$ and $\sigma_{\text {in }}$ were their respective standard deviations.

\section{RESULTS}

The results of the simulated cyst phantom obtained using the $\mathrm{C}-1$ virtual sources configurations and $\mathrm{S}-1$ diverging wave strategy is shown Fig.3 The images Fig. 3a shows the results without motion compensation containing noticeable motion artifacts and resulting in blurry and low image quality. The image Fig. $3 \mathrm{~b}$ depicts the results with motion compensation qualitatively showing the restored motion and image quality. 
Quantitatively, the CNRs and CRs of the nine strategies are summarized in Fig. 4. The bars in blue/red colors indicate the results without/with MoCo, respectively. Concerning the CR, without motion compensation, the diverging wave strategy $\mathrm{S}-2$ shows higher $\mathrm{CR}$ for the three virtual sources configuration ( C-1, C-2, and C-3). However, when motion compensation is applied, the diverging wave strategy S-1 shows slightly higher $\mathrm{CR}$ for the three virtual source configurations. Moreover, the virtual sources configuration C-3 (corresponding to smaller sub-aperture) give slightly higher $\mathrm{CNR}$ and $\mathrm{CR}$ than $\mathrm{C}-2$ and $\mathrm{C} 1$.

Overall, the motion compensation algorithm increased, on average, for the S-1, S-2, and S-3, the CNR/SNR of 3/10.2, 2.9/9.8 and, 1.8/9.3, in $\mathrm{dB}$, respectively.

\section{DisCUSSION / CONCLUSIONS}

In this work, the influence of the MoCo approach on three different diverging waves strategies with three different configurations of virtual sources has been quantitavely conducted in simulation. First, the positions of the virtual sources have been formalized according to the chosen diverging waves strategy and the diverging waves opening angle. Then the approaches were compared quantitatively by estimated the CNR and CR. Even if the nine strategies gave similar results, the diverging waves strategies S-1 combined with the $\mathrm{C}-1$ virtual sources configuration gave slightly better $\mathrm{CNR}$ and $\mathrm{CR}$. The results confirmed that MoCo increased both the CNR and the CR for each case. In average, the $\mathrm{CNR} / \mathrm{CR}$ has been increased of $+3 / 10.2,2.9 / 9.8$ and, 1.8/9.3, in $\mathrm{dB}$, for $\mathrm{S}-1 \mathrm{~S}-2$ and $\mathrm{S}-3$ strategy, respectively. The simulations have been carried out only one time with only one axial velocity for each case, which is the main limitation of this study. Moreover only the axial component of the velocity has been estimated and compensated, which will be insufficient in case of fast displacement in the lateral direction. More simulation results and experimental validation of the $3 \mathrm{D}$ motion compensation strategy has now to be evaluated.

ACKNOWLEDGMENT

This work was supported by the LABEX PRIMES (ANR-11LABX-0063) and the LABEX CELYA (ANR-10-LABX0060) of the Universite de Lyon, within the programme ' Investissements d'Avenir' (ANR-11-IDEX-0007), operated by the French National Research Agency (ANR).

\section{REFERENCES}

[1] G. Montaldo, M. Tanter, J. Bercoff, N. Benech, and M. Fink, "Coherent plane-wave compounding for very high frame rate ultrasonography and transient elastography," IEEE Trans. Ultrason. Ferroelectr. Freq. Control, vol. 56, no. 3, pp. 489-506, Mar. 2009.

[2] J. Udesen, F. Gran, K. L. Hansen, J. A. Jensen, C. Thomsen, and M. B. Nielsen, "High frame-rate blood vector velocity imaging using plane waves: simulations and preliminary experiments," IEEE Trans. Ultrason. Ferroelectr. Freq. Control, vol. 55, no. 8, pp. 1729-1743, Aug. 2008.

[3] L. Tong, H. Gao, and J. D'hooge, "Multi-transmit beam forming for fast cardiac imaging-a simulation study," IEEE Trans. Ultrason. Ferroelectr. Freq. Control, vol. 60, no. 8, pp. 1719-1731, Aug. 2013.

[4] J. Provost et al., "3D ultrafast ultrasound imaging in vivo," Phys. Med. Biol., vol. 59, no. 19, pp. L1-L13, Oct. 2014.

[5] S. Salles, H. Liebgott, D. Garcia, and D. Vray, "Real time 3D US-tagging combined with 3D phasebased motion estimation," 2013, pp. 585-588.

[6] E. Badescu, D. Bujoreanu, L. Petrusca, D. Friboulet, and H. Liebgott, "Multi-line transmission for 3D ultrasound imaging: An experimental study," in 2017 IEEE International Ultrasonics Symposium (IUS), Washington, DC, 2017, pp. 1-4.

[7] J. Poree, D. Posada, A. Hodzic, F. Tournoux, G. Cloutier, and D. Garcia, "High-Frame-Rate Echocardiography Using Coherent Compounding With Doppler-Based Motion-Compensation," IEEE Trans. Med. Imaging, vol. 35, no. 7, pp. 1647-1657, Jul. 2016.

[8] B. Denarie et al., "Coherent Plane Wave Compounding for Very High Frame Rate Ultrasonography of Rapidly Moving Targets," IEEE Trans. Med. Imaging, vol. 32, no. 7, pp. 1265-1276, Jul. 2013.

[9] K. L. Gammelmark and J. A. Jensen, "2-D tissue motion compensation of synthetic transmit aperture images," IEEE Trans. Ultrason. Ferroelectr. Freq. Control, vol. 61, no. 4, pp. 594-610, Apr. 2014.

[10] Y. Chen, J. D'hooge, and J. Luo, "Doppler-Based Motion Compensation Strategies for 3-D Diverging Wave Compounding and Multiplane-Transmit Beamforming: A Simulation Study," IEEE Trans. Ultrason. Ferroelectr. Freq. Control, vol. 65, no. 9, pp. 1631-1642, Sep. 2018.

[11] P. Joos et al., "High-frame-rate 3-D echocardiography based on motion compensation: An in vitro evaluation," in 2017 IEEE International Ultrasonics Symposium (IUS), Washington, DC, USA, 2017, pp. $1-4$.

[12] J. A. Jensen, "FIELD: A Program for Simulating Ultrasound Systems," in 10TH NORDICBALTIC CONFERENCE ON BIOMEDICAL IMAGING, VOL. 4, SUPPLEMENT 1, PART 1:351-353, 1996, pp. 351-353.

[13] J. A. Jensen and N. B. Svendsen, "Calculation of pressure fields from arbitrarily shaped, apodized, and excited ultrasound transducers," IEEE Trans. Ultrason. Ferroelectr. Freq. Control, vol. 39, no. 2, pp. 262-267, Mar. 1992. 